\title{
Sending the Signal: Muscle Glycogen Availability as a Regulator of Training Adaptation
}

\author{
John A. Hawley
}

\begin{abstract}
Exercise training-induced adaptations in human skeletal muscle are largely determined by the mode, volume, intensity and frequency of the training stimulus. However, a growing body of evidence demonstrates that the availability of endogenous and exogenous macronutrients can modify multiple intramuscular responses to both endurance- and resistance-based exercise. Acutely manipulating substrate availability (by altering diet composition and/or timing of meals) rapidly alters the concentration of blood-borne substrates and hormones that modulate several receptor-mediated signaling pathways. The release of cytokines and growth factors from contracting skeletal muscle also stimulates cell surface receptors and activates many intracellular signaling cascades. These local and systemic factors cause marked perturbations in the storage profile of skeletal muscle (and other insulin-sensitive tissues) that, in turn, exert pronounced effects on resting fuel metabolism and patterns of fuel utilization during exercise. When repeated over weeks and months, such nutrient-exercise interactions have the potential to alter numerous adaptive processes in skeletal muscle that ultimately drive the phenotypespecific variability observed between individuals. One strategy that augments endurance-training adaptation is commencing exercise with low muscle glycogen concentration ("train-low"). The amplified training response observed with low endogenous carbohydrate availability is likely regulated by enhanced activation of key cell signalling kinases (e.g., AMPK, p38MAPK), transcription factors (e.g., p53, PPARס) and transcriptional co-activators (e.g., PGC-1 $\alpha$ ), such that a coordinated up-regulation of both the nuclear and mitochondrial genomes occurs. This chapter provides a contemporary perspective of our understanding of the molecular and cellular events that take place in skeletal muscle in response to exercise commenced after alterations in nutrient availability and discusses how the ensuing hor-
\end{abstract}

\footnotetext{
J. A. Hawley $(\square)$

Mary MacKillop Institute for Health Research, Australian Catholic University, Melbourne, VIC, Australia

Research Institute for Sport and Exercise Sciences, Liverpool John Moores University, Liverpool, UK

e-mail: john.hawley@acu.edu.au
} 
monal milieu interacts with specific contractile stimulus to modulate many of the acute responses to exercise, thereby potentially promoting or inhibiting subsequent training adaptation.

\section{Introduction}

Classic studies conducted in the late nineteenth and early twentieth century determined that the fuels that supported contracting skeletal muscle during continuous exercise lasting longer than several minutes were intra- and extra-muscular carbohydrate and lipid substrates, with only a minor contribution from amino acids (for review, see Hawley et al. 2015). During this time, it was also demonstrated that manipulation of the macronutrient content of the preceding diet resulted in marked changes in the time for which exercise at a fixed, submaximal power output could be sustained; furthermore, subjects perceived exercise to be easier after several days of a high carbohydrate diet than when the preceding diet was low in carbohydrate and high in fat (Hawley et al. 2015). In the mid twentieth century, a large body of work, mainly undertaken in laboratories in Europe, extended the earlier observations of the importance of carbohydrate-based fuels for prolonged, intense exercise.

The period from 1960 up until the late 1980s has been termed the "classical period" of exercise biochemistry (Brooks and Mercier 1994). With remarkable foresight, Goldstein (1961) proposed that a "humoral factor" was liberated by muscle during contraction that "activates the glucose transport system" and helps "regulate carbohydrate metabolism" in what was later to become known as "muscle cross talk." At the beginning of the 1960s, little was known about the regulation of mitochondrial biogenesis in skeletal muscle in response to exercise training. However, seminal work by Holloszy (1967) heralded a major advance in unravelling some of the cellular events controlling muscle bioenergetics in response to exercise. It was during this time that the percutaneous needle biopsy technique was introduced into exercise biochemistry (Bergström and Hultman 1966), making it possible to conduct invasive studies and determine the impact of training, diet, and other manipulations on selected metabolic characteristics. Studies conducted in labs in Europe and North America advanced our understanding of the impact of training and diet manipulations on muscle substrate stores, the interactive effects of exercise and orally administered carbohydrate on muscle glucose kinetics and exercise capacity, and the protein synthetic response to exercise (Hawley et al. 2015; Brooks and Mercier 1994). New insights into the influence of substrate availability on the hormonal response to prolonged exercise were also advanced in this period (Galbo et al. 1979). At the same time, major progress in our knowledge of how exercise activates cellular, molecular, and biochemical pathways with regulatory roles in training response adaptation took place, and skeletal muscle was confirmed to have endocrine-like effects, releasing cytokines and other peptides to orchestrate interorgan "cross talk" (Pedersen et al. 2003). 
During the past two decades, exercise training-induced adaptations in skeletal muscle have been shown to be the result of the cumulative effects of the acute responses to repeated, single exercise bouts (Perry et al. 2010), with the rapid and transient changes in gene transcription following a single bout of exercise leading to chronic effects on protein expression when repeated over time. However, only recently have we begun to appreciate the impact that alterations in substrate availability have on modifying training responses-adaptations. While substrates like carbohydrate and fat were viewed merely as energy stores for contracting muscles, we are now beginning to understand that fuel availability per se is a crucial regulator of many biochemical and cellular signaling events with roles in mitochondrial biogenesis, autophagy, health and performance. This chapter will provide a contemporary perspective of our understanding of the regulation of substrate metabolism; the molecular and cellular events that take place in skeletal muscle in response to exercise commenced after alterations in nutrient availability; and how the ensuing hormonal milieu interacts with specific contractile stimulus to modulate many of the acute responses to exercise, thereby potentially promoting or inhibiting subsequent training adaptation.

\section{Fuels for the Fire: Patterns of Skeletal Muscle Fuel Utilization}

Both carbohydrate- (muscle and liver glycogen, blood glucose, muscle and liver lactate) and fat- (adipose and intramuscular triglycerides, blood-borne free fatty acids and triglycerides) based fuels are important substrates for oxidative phosphorylation and energy production in skeletal muscle (Brooks and Mercier 1994). Compared to the finite stores of carbohydrate, endogenous lipid stores in humans are plentiful and represent a potentially unlimited source of fuel for skeletal muscle metabolism during aerobic exercise. However, fatty acid (FA) oxidation by muscle is limited, at least during the power outputs sustained by athletes during training and competition (Hawley and Leckey 2015). Unlike the oxidation of carbohydrate, which is closely geared to the energy requirements of the working muscle, there are no mechanisms for matching the availability and utilization of FA to the rate of energy expenditure (Holloszy et al. 1988). However, the oxidation of any one fuel or another at rest or during exercise does not occur in isolation and is typically the result of the integration of many metabolic signals involving exogenous and endogenous substrate availability, the prevailing hormonal milieu as well as muscle and whole-body energetic demands.

Both carbohydrate- and fat-based fuels are oxidized at rest to provide the energy required for basal metabolic processes in skeletal muscle, with a reciprocal relationship between the utilization of carbohydrate and fat: fuel shifts occurring at rest are largely driven by the availability of substrates and the concomitant hormonal environment (i.e., plasma glucose, insulin, FFA and glucagon concentrations) in the face of a generally unchanged metabolic demand. For example, increasing blood glucose availability by feeding of a carbohydrate-rich meal 
increases the uptake and oxidation of glucose into skeletal muscle while concomitantly decreasing the oxidation of fat; but overall, there is little change in resting metabolic rate. The decreased rate of FA oxidation at rest is largely attributable to a carbohydrate feeding-induced rise in insulin concentration that impairs lipolysis (i.e., the rate of appearance [Ra] of FFA into the systemic circulation), an effect that can persist for up to four hours after a carbohydrate-rich meal (Bonadonna et al. 1990).

The concept of a reciprocal relationship between fat and carbohydrate oxidation, at least in resting muscle, stemmed from the work of Randle and colleagues in the 1960s (Randle et al. 1964). Their experiments demonstrated that increased lipid availability [i.e., circulating plasma free fatty acids (FFA)] increased FA oxidation and decreased carbohydrate oxidation in muscle and that it was the increase in fat availability that resulted in key intracellular perturbations, including increased contents of muscle acetyl-coenzyme A (CoA), citrate, and glucose-6-phosphate: lipidinduced changes at these key regulatory sites decreased muscle carbohydrate metabolism. The results of these early studies, which subsequently formed the bases of the "glucose-fatty acid cycle" hypothesis (Randle 1986), were obtained by comparing supra-physiological levels of FFA while glucose concentration was "clamped." The prevailing paradigm was that carbohydrate-based substrates were the muscle's "default" fuel but that increased FA availability could "switch" muscle fuel use and down-regulate carbohydrate metabolism. While the "glucose-fatty acid cycle" proposed by Randle and coworkers (Randle et al. 1964) explained the mechanisms responsible for the down regulation of carbohydrate metabolism in the face of high FA availability, these researchers failed to perform the control or "crossover" experiments, in which FA availability was held constant but glucose levels were increased. This, apparently, was because they steadfastly believed that there was little regulation of fat metabolism in skeletal muscle at rest (or presumably during exercise) and that the key determinant of the rate of FA oxidation was simply a function of the availability of fat and its regulation at the level of the mitochondrial membranes through the carnitine-palmitoyl transferase (CPT) complex (Randle et al. 1964).

While there are situations in which the glucose-fatty acid cycle operates, a series of elegant studies conducted in the late 1990s clearly demonstrated that carbohydrate availability directly regulates fat oxidation at rest and during exercise (Coyle et al. 1997; Horowitz et al. 1997; Romijn et al. 1995). Coyle et al. (1997) demonstrated that a carbohydrate-rich meal that induced hyperglycemia and hyperinsulinemia increased glycolytic flux and directly reduced rates of FA oxidation during low-intensity exercise. Carbohydrate feeding reduced the Ra of plasma FFA by $\sim 35 \%$, resulting in a reduction in both plasma FFA and intramuscular triglyceride oxidation, suggesting a coordinated effect of increased glucose availability on adipose tissue and muscle. These observations indicate that glucose can directly regulate fat oxidation during exercise and that the classic "glucose-fatty acid cycle" proposed by Randle and colleagues (Randle 1986; Randle et al. 1964), in which fat availability alone regulates rates of FA oxidation, is an overly simplistic view of fuel regulation. 
The concept that altering substrate availability can modify the proportions of carbohydrate and fat utilized by working muscle is certainly not new (Hawley et al. 2015). However, during the past decade, data from several independent laboratories has provided evidence that commencing selected exercise sessions under conditions of reduced carbohydrate availability can promote training-induced adaptations in human skeletal muscle to a greater magnitude than if the same sessions were commenced with normal or high glycogen levels.

\section{Sending the Signal: The Training Response-Adaptation}

The goals of endurance exercise training are to induce an array of physiological and metabolic adaptations that enable an individual to increase the rate of energy production from both aerobic and oxygen-independent pathways, maintain tighter metabolic control (i.e., match ATP production with ATP hydrolysis), minimize cellular perturbations, increase efficiency of motion, and improve the capacity of the trained musculature to resist fatigue (Hawley 2002). The mechanisms and metabolic signals by which active muscle senses homeostatic perturbations and then translates them into improved function has been a topic of intense research for several decades (for review, see Perry and Hawley 2017). It is now accepted that a variety of cellular disruptions takes place at the onset of exercise, including (but not limited to) increased cytoplasmic free $\left[\mathrm{Ca}^{2+}\right]$, increased free AMP (AMPf) and an increased ADP/ATP ratio, reduced creatine phosphate and glycogen levels, increased FA concentrations and reactive oxygen/nitrogen species (ROS/RNS), acidosis and altered redox state, including [NAD/NADH] (Hawley et al. 2014; Perry and Hawley 2017). Within the context of metabolic homeostasis, an array of regulatory networks is stimulated that sustain rates of ATP synthesis over time through the activation of rate-limiting enzymes controlling carbohydrate and fat catabolism. A long-standing question in exercise biology is how do these acute disruptions in cellular signals that maintain energy supply also stimulate long-term adaptive processes that improve the ability of muscle to sustain a future contractile challenge?

A key component of improved "muscle fitness" following exercise training is biogenesis of mitochondria in skeletal muscle. A comprehensive discussion of this topic is beyond the scope of this chapter and the reader is referred to recent reviews (Hood et al. 2016; Perry and Hawley 2017). In brief, the molecular bases of skeletal muscle adaptations to an endurance exercise stimulus requires increased expression and/or activity of key mitochondrial proteins mediated by an array of intra-cellular signaling events, pre- and post-transcriptional processes, regulation of translation and protein expression, and modulation of protein (enzyme) activities and/or intracellular localization. There are multiple (and often redundant) stimuli associated with endurance exercise adaptations and numerous signaling kinases that respond to contractile stimuli, with numerous downstream pathways that are targets of these kinases (Egan et al. 2016; Hawley et al. 2014). A major advance in unraveling the cellular events that promote mitochondrial biogenesis was the discovery of the 
peroxisome-proliferator-activated receptor $\gamma$ coactivator $\alpha$ (PGC-1 $\alpha$ ), an inducible coactivator that regulates the coordinated expression of mitochondrial proteins encoded in the nuclear and mitochondrial genomes (Lin et al. 2002). A critical feature of the PGC- $1 \alpha$ coactivators is that they interact with many different transcription factors to activate distinct biological programs in several tissues (Lin et al. 2002). Certainly in skeletal muscle, PGC-1 $\alpha$ has emerged as a key regulator of mitochondrial biogenesis responsive to the prevailing contractile activity.

The AMPK and p38 MAPK are two other important signaling cascades that converge upon the regulation of PGC- $1 \alpha$ and consequently the regulation of mitochondrial biogenesis. The AMPK induces mitochondrial biogenesis partly by directly phosphorylating and activating PGC-1 $\alpha$ (Jager et al. 2007) but also by phosphorylating the transcriptional repressor HDAC5, which relieves inhibition of the transcription factor myocyte enhancer factor 2 (MEF2), a known regulator of PGC-1 $\alpha$ (McGee and Hargreaves 2010). p38 MAPK phosphorylates and activates PGC-1 $\alpha$ (Puigserver et al. 2001) and also increases PGC-1 $\alpha$ expression by phosphorylating the transcription factor ATF-2, which in turn increases PGC- $1 \alpha$ protein abundance by binding to and activating the CREB site on the PGC- $1 \alpha$ promoter (Akimoto et al. 2005). Another transcription factor involved in exercise-induced mitochondrial biogenesis in skeletal muscle and presumably activated by AMPK and/or p38 MAPK is the tumor suppressor protein p53. p53 knockout mice display reduced endurance exercise capacity compared with wild-type mice, along with reduced subsarcolemmal and inter-myofibrillar mitochondrial content and PGC-1 $\alpha$ expression. p53 may also regulate exercise-induced mitochondrial biogenesis through interactions with TFAM in the mitochondria, where it functions to co-ordinate regulation of the mitochondrial genome (Saleem et al. 2011).

\section{Train-Low, Compete High: A Novel Strategy to Augment Training Adaptation}

Skeletal muscle glycogen availability exerts a regulatory effect on many cellular processes. As discussed, one protein with a fundamental role in monitoring cellular energy status is the AMPK. The recent discovery of glycogen-binding sites on the AMPK $\beta$-subunits (McBride et al. 2009) has led to the hypothesis that this regulatory domain may also allow AMPK to act as a sensor of endogenous glycogen stores (McBride and Hardie 2009). In this scenario, the glycogen-binding domains act as sensors, enabling AMPK to gauge the state of cellular glycogen, increasing AMPK activity when stores are low and decreasing the signal when stores are replete or elevated. The first experimental evidence from human skeletal muscle to provide indirect support for this hypothesis was undertaken by Wojtaszewski et al. (2003). These workers measured muscle-signaling responses and substrate utilization in well-trained males under conditions in which a standardized bout of exercise ( $1 \mathrm{~h}$ at $70 \%$ peak oxygen consumption) was commenced with either low ( $\sim 160 \mathrm{mmol} / \mathrm{kg}$ dry wt $)$ or high $(\sim 900 \mathrm{mmol} / \mathrm{kg}$ dry wt) muscle glycogen concentration. At rest, AMPK activity and acetyl-CoA 
carboxylase- $\beta$ (ACC- $\beta$ ) Ser ${ }^{221}$ phosphorylation were lower in glycogen-loaded compared with glycogen-depleted muscles. Of note was the finding that concentrations of creatine phosphate and adenine nucleotides in resting muscles were similar despite large differences in muscle glycogen status, suggesting that fuel-dependent mechanisms independent of energy status might regulate AMPK signaling. AMPK- $\alpha 1$ and $\alpha 2$ activity and ACC- $\beta$ Ser ${ }^{221}$ phosphorylation were activated to a greater extent when exercise was commenced with low compared to high glycogen levels. Subsequently, Steinberg et al. (2006) reported that commencing exercise with low muscle glycogen availability increased AMPK- $\alpha 2$ nuclear protein abundance. The results of these studies make it tempting to ascribe a direct mechanistic link between glycogen availability and AMPK activation. However, manipulating resting muscle glycogen content in humans also alters the concentrations of circulating blood metabolites (FFAs, catecholamines). These systemic factors are likely to "fine-tune" the signaling responses observed when exercise is commenced with low glycogen stores.

The early observations of increased AMPK activation when exercise is commenced with low muscle glycogen availability coupled with the putative role of the AMPK in many of the metabolic adaptations of skeletal muscle to exercise-training (Jørgensen et al. 2006; Pilegaard et al. 2002) were the bases of a hypothesis formulated by Hansen et al. (2005), namely that chronic exercise training undertaken with low muscle glycogen concentration would improve training adaptation to a greater extent compared to when all sessions were commenced with high glycogen availability. This training protocol was termed "train-low, compete-high." In a pioneering study, Hansen et al. (2005) used an experimental design in which the right and left legs of the same (untrained) subject performed the same amount of total work during a 10-week intervention period, albeit with different pre-exercise muscle glycogen content for half of the workouts. In agreement with their original hypothesis, they reported that markers of "training adaptation" (i.e., resting muscle glycogen content, the maximal activity of citrate synthase and performance time to exhaustion) were all enhanced to a greater extent in the leg that commenced selected training sessions with low compared with high muscle glycogen content (Hansen et al. 2005). The results of this study were groundbreaking, and the term "train-low, compete-high" rapidly spread among the athletic and scientific communities to describe this novel approach to training. However, the question of whether athletes with a history of endurance training would benefit to the same extent as previously untrained individuals when replacing a portion of their training program with "low glycogen" workouts could not be answered from the original study of Hansen et al. (2005).

In a follow-up investigation, Yeo et al. (2008) investigated the "train-low" paradigm during a 3-week intervention in well-trained endurance subjects: these researchers hypothesized that competitive athletes would have maximized their training adaptation, and further gains, even in the face of training-low, would be trivial. Yeo et al. (2008) imposed a training protocol in which nine intense interval-training sessions during the 3-week training block were commenced when glycogen stores were lowered by $\sim 50 \%$ (through prior exercise) or were replete. Importantly, during these sessions, the work rate (power output, W) was not "clamped" as in the study of Hansen et al. (2005); rather athletes were asked to 
exercise as hard as they could and produce as much total work as possible. As expected, when athletes commenced training with low compared with normal glycogen availability, their maximal self-selected power output was significantly lower (an average of 7\%). The remarkable finding, however, was that, despite a lower "training impulse" to the exercising musculature, resting muscle glycogen concentration, the maximal activities of citrate synthase and $\beta$-hydroxyacyl-CoAdehydrogenase and the total protein content of cytochrome $c$ oxidase subunit IV were higher (compared to pre-training values) in individuals who commenced interval training sessions with low muscle glycogen content. Despite these augmented training adaptations, which would be expected to enhance athletic performance, and in contrast to the findings of Hansen et al. (2005), there was no difference between the two treatment conditions in a sport-specific cycling time-trial (Yeo et al. (2008).

The precise mechanisms underlying an enhanced adaptive skeletal muscle response when training sessions were commenced with lowered glycogen availability were not investigated during these investigations (Hansen et al. 2005; Yeo et al. 2008). However, from earlier work, MAPK pathways have been implicated as possible signaling mechanisms involved in the regulation of exercise/nutrient adaptations. Chan et al. (2004) were the first to demonstrate that alterations in nutrient availability that reduced muscle glycogen content led to an increased phosphorylation of nuclear p38 MAPK in human skeletal muscle in response to moderateintensity exercise. However, others have found little change in either the phosphorylation state of this kinase or one of its downstream targets (activating transcription factor 2) after a bout of intense cycling commenced with either low or normal muscle glycogen content (Yeo et al. 2010).

It is important to note that altering carbohydrate availability (i.e., lowering muscle glycogen content) has reciprocal and pronounced effects on lipid availability. Indeed it seems entirely plausible that increases in skeletal muscle mitochondria capacity could be further enhanced if training were performed under conditions that further elevated the levels of circulating FFAs. To test this hypothesis, Fillmore et al. (2010) determined whether a combination of chronic chemical activation of AMPK and high-fat availability (a low-CHO, high-fat diet) would have an additive effect on skeletal muscle mitochondria markers in a rodent model. They treated male Wistar rats with a high-fat diet, with injections of AICAR (an AMPK activator), or with a high-fat diet combined with AICAR injections for 6 weeks. Compared to either AICAR or high-fat feeding alone, AICAR treatment combined with high fat availability had an additive effect on markers of oxidative capacity (i.e., the citric acid cycle and electron transport chain) as well as transcriptional regulation. Specifically, long-chain acyl-CoA dehydrogenase, cytochrome $c$, PGC-1 $\alpha$ protein, as well citrate synthase and $\beta$-hydroxyacyl-CoA dehydrogenase activity were all increased by a greater magnitude in the AICAR plus high-fat treatment. While the mechanism(s) responsible for the additive increase in mitochondrial content observed with chronic activation of AMPK and elevating circulating FFAs could not be directly determined, Fillmore et al. (2010) observed an synergistic effect of chronic AMPK activation and high-fat feeding on PGC-1 $\alpha$ protein abundance and elevations in PGC-1 $\alpha$ mRNA in response to chronic AMPK activation (but not by 
high-fat feeding). They concluded that the additive increase in PGC- $1 \alpha$ protein abundance was a combined effect of high-fat feeding-induced posttranscriptional and AMPK-dependent transcriptional increases in PGC-1 $\alpha$ protein expression. However, it should be noted that, when post- exercise increases in FA availability are abolished by administration of acipimox, a pharmacological inhibitor of lipolysis, the normal exercise-induced increase in mRNA abundance of selected metabolic genes (i.e., PDK4 and PGC-1 $\alpha$ ) persists (Tunstall et al. 2007). These data clearly demonstrate that the increase in circulating FFA concentrations observed during the later stages of exercise and subsequent recovery are not essential to induce skeletal muscle mRNA expression of several proteins involved in regulating muscle metabolism and training adaptation.

\section{Evolution of a Paradigm: Train-High, Sleep-Low}

The original "train low" protocol proposed by Hansen et al. (2005) and subsequently investigated by others (Hulston et al. 2010; Yeo et al. 2008) employed a twice-a-day training protocol in which the second exercise session was undertaken with low glycogen availability. As noted, a direct consequence of this strategy was that the maximal self-selected training intensity of the second session was substantially reduced when it was commenced with low compared with normal glycogen levels. Such an outcome is counter-intuitive for the preparation of competitive athletes for whom high-intensity workouts are a critical component of any periodized training program (Hawley and Burke 2010). Furthermore, it could be argued that training twice each day with workouts undertaken in close proximity underpinned part of the augmented training adaptation compared to once-a-day workouts. Against this background, we formulated a novel approach in which we can prolong the duration of low carbohydrate availability, thereby potentially enhancing and extending the time course of transcriptional activation of metabolic genes and their target proteins, while simultaneously conserving the training intensity of the initial session and hence the "training impulse" to the working muscles (Lane et al. 2015). We have termed this strategy "train-high, sleep-low." In this protocol, we periodized the timing of nutrient intake such that athletes performed an evening bout of high-intensity training with high carbohydrate availability, then restricted carbohydrate intake so that they slept with low carbohydrate availability before undertaking a prolonged exercise bout $(120 \mathrm{~min})$ in the fasted state the following morning. In our acute model (Lane et al. 2015), we found that, when feeding was withheld overnight and subjects slept with reduced carbohydrate availability, AMPKThr172, p38MAPKThr180/Tyr182, and p-ACCSer79 were upregulated to a greater extent the following morning, compared with when subjects were fed a high- carbohydrate meal the prior evening. We also showed that, when a second, prolonged steady-state training session was commenced after "sleeping low," the expression of selected genes and abundance of phosphorylated signaling proteins with putative roles in lipid oxidation and transport were higher compared with when a post-exercise meal was 
consumed and glycogen availability was partially restored (Lane et al. 2015). Importantly, subsequent chronic interventions utilizing the "train-high, sleep-low" approach over 1-3 weeks showed clear benefits to performance (Marquet et al. 2016a, b).

\section{Summary and Directions for Future Research}

Independent of prior training status, short-term (3- to 10-week) training programs in which some of the workouts are commenced with either low muscle glycogen availability (or low exogenous carbohydrate availability) augment training adaptation (i.e., they increase the maximal activities of selected enzymes involved in carbohydrate and/or lipid metabolism and promote mitochondrial biogenesis) to a greater extent than when all workouts are undertaken with normal or elevated glycogen stores. One aspect that is unclear from the present literature is the absolute levels of glycogen depletion needed to potentiate the effect of the training stimulus on outcomes such as mitochondrial biogenesis, or the length of time periodic low-glycogen training needs to be undertaken to demonstrate functional changes to training and/or performance outcomes. To answer such questions, a complex series of studies would need to be undertaken that systematically 'titrate' muscle glycogen levels and determine subsequent cellular (and performance) response to a standardized training regimen. A major problem for the basic scientist trying to unravel potential mechanism(s) underlying the benefit to training adaptation with reduced muscle glycogen availability is the fact that carbohydrate restriction has reciprocal and pronounced effects on lipid availability. To address this question, it will be necessary to study human subjects while they are consuming isoenergetic low carbohydrate diets in combination with either high protein or fat while undertaking a supervised exercise training protocol. Such experiments are currently being undertaken in our lab. Invasive measures of metabolism (i.e., markers of skeletal muscle mitochondrial biogenesis and respiration) are necessary to determine if it is high fat or low carbohydrate availability that drives the augmented training response. What is clear, however, is that in addition to their role as energy substrates, endogenous fuel stores and the concomitant changes in circulating hormones and metabolites can act as potent signaling intermediates to modify the normal responses to contractile activity, thereby augmenting training adaptation and ultimately driving some of the phenotypical changes observed with chronic exercise.

\section{References}

Akimoto T, Pohnert SC, Li P, Zhang M, Gumbs C, Rosenberg PB, Williams RS, Yan Z (2005) Exercise stimulates Pgc-1alpha transcription in skeletal muscle through activation of the p38 MAPK pathway. J Biol Chem 280:19587-19593 
Bergström J, Hultman E (1966) Muscle glycogen synthesis after exercise: an enhancing factor localized to the muscle cells in man. Nature 210:309-310

Bonadonna RC, Groop LC, Zych K, Shank KM, DeFronzo RA (1990) Dose-dependent effect of insulin on plasma free fatty acid turnover and oxidation in humans. Am J Physiol Endocrinol Metab 259:E736-E750

Brooks GA, Mercier J (1994) Balance of carbohydrate and lipid utilization during exercise: the "crossover" concept. J Appl Physiol 76:2253-2261

Chan MH, McGee SL, Watt MJ, Hargreaves M, Febbraio MA (2004) Altering dietary nutrient intake that reduces glycogen content leads to phosphorylation of nuclear p38 MAP kinase in human skeletal muscle: association with IL-6 gene transcription during contraction. FASEB J 18:1785-1787

Coyle EF, Jeukendrup AE, Wagenmakers AJ, Saris WH (1997) Fatty acid oxidation is directly regulated by carbohydrate metabolism during exercise. Am J Physiol Endocrinol Metab 273:E268-E275

Egan B, Hawley JA, Zierath JR (2016) SnapShot: exercise metabolism. Cell Metab 24:342-342.e1

Fillmore N, Jacobs DL, Mills DB, Winder WW, Hancock CR (2010) Chronic AMP-activated protein kinase activation and a high-fat diet have an additive effect on mitochondria in rat skeletal muscle. J Appl Physiol 109:511-520

Galbo H, Holst JJ, Christensen NJ (1979) The effect of different diets and of insulin on the hormonal response to prolonged exercise. Acta Physiol Scand 107:19-32

Goldstein MS (1961) Humoral nature of the hypoglycemic factor of muscular work. Diabetes 10:232-234

Hansen AK, Fischer CP, Plomgaard P, Andersen JL, Saltin B, Pedersen BK (2005) Skeletal muscle adaptation: training twice every second day vs. training once daily. J Appl Physiol 98:93-99

Hawley JA (2002) Adaptations of skeletal muscle to prolonged, intense endurance training. Clin Exp Pharmacol Physiol 29:218-222

Hawley JA, Burke LM (2010) Carbohydrate availability and training adaptation: effects on cell metabolism. Exerc Sport Sci Rev 38:152-160

Hawley JA, Leckey JJ (2015) Carbohydrate dependence during prolonged, intense endurance exercise. Sports Med 45(Suppl 1):S5-12

Hawley JA, Hargreaves M, Joyner MJ, Zierath JR (2014) Integrative biology of exercise. Cell 159:738-749

Hawley JA, Maughan RJ, Hargreaves M (2015) Exercise metabolism: historical perspective. Cell Metab 22:12-17

Holloszy JO (1967) Biochemical adaptations in muscle. Effects of exercise on mitochondrial -oxygen uptake and respiratory enzyme activity in skeletal muscle. J Biol Chem 242:2278-2282

Holloszy JO, Kohrt WM, Hansen PA (1988) The regulation of carbohydrate and fat metabolism during and after exercise. Front Biosci 3:D1011-D1027

Hood DA, Tryon LD, Carter HN, Kim Y, Chen CC (2016) Unravelling the mechanisms regulating muscle mitochondrial biogenesis. Biochem J 473:2295-2314

Horowitz JF, Mora-Rodriguez R, Byerley LO, Coyle EF (1997) Lipolytic suppression following carbohydrate ingestion limits fat oxidation during exercise. Am J Physiol 273:E768-E775

Hulston CJ, Venables MC, Mann CH, Martin C, Philp A, Baar K, Jeukendrup AE (2010) Training with low muscle glycogen enhances fat metabolism in well-trained cyclists. Med Sci Sports Exerc 42:2046-2055

Jager S, Handschin C, St-Pierre J, Spiegelman BM (2007) AMP-activated protein kinase (AMPK) action in skeletal muscle via direct phosphorylation of PGC-1a. Proc Natl Acad Sci USA 104:12017-12022

Jørgensen SB, Richter EA, Wojtaszewski JFP (2006) Role of AMPK in skeletal muscle metabolic regulation and adaptation in relation to exercise. J Physiol 574:17-31 
Lane SC, Camera DM, Lassiter DG, Areta JL, Bird SR, Yeo WK, Jeacocke NA, Krook A, Zierath JR, Burke LM, Hawley JA (2015) Effects of sleeping with reduced carbohydrate availability on acute training responses. J Appl Physiol 119:643-655

Lin J, Wu H, Tarr PT, Zhang CY, Wu Z, Boss O, Michael LF, Puigserver P, Isotani E, Olson EN, Lowell BB, Bassel-Duby R, Spiegelman BM (2002) Transcriptional co-activator PGC-1 alpha drives the formation of slow-twitch muscle fibres. Nature 418:797-801

Marquet LA, Brisswalter J, Louis J, Tiollier E, Burke LM, Hawley JA, Hausswirth C (2016a) Enhanced endurance performance by periodization of carbohydrate intake: "sleep low" strategy. Med Sci Sports Exerc 48:663-672

Marquet LA, Hausswirth C, Molle O, Hawley JA, Burke LM, Tiollier E, Brisswalter J (2016b) Periodization of carbohydrate intake: short-term effect on performance. Nutrients 8(12):E755

McBride A, Hardie DG (2009) AMP-activated protein kinase - a sensor of glycogen as well as AMP and ATP? Acta Physiol (Oxf) 196:99-113

McBride A, Ghilagaber S, Nikolaev A, Hardie DG (2009) The glycogen-binding domain on the AMPK beta subunit allows the kinase to act as a glycogen sensor. Cell Metab 9:23-34

McGee SL, Hargreaves M (2010) AMPK-mediated regulation of transcription in skeletal muscle. Clin Sci 118:507-518

Pedersen BK, Steensberg A, Fischer C, Keller C, Keller P, Plomgaard P, Febbraio M, Saltin B (2003) Searching for the exercise factor: is IL-6 a candidate? J Muscle Res Cell Motil 24:113-119

Perry CG, Hawley JA (2017) Molecular basis of exercise-induced skeletal muscle mitochondrial biogenesis: Historical advances, current knowledge, and future challenges. Cold Spring Harb Perspect Biol. pii: a029686

Perry CG, Lally J, Holloway GP, Heigenhauser GJ, Bonen A, Spriet LL (2010) Repeated transient mRNA bursts precede increases in transcriptional and mitochondrial proteins during training in human skeletal muscle. J Physiol 588:4795-4810

Pilegaard H, Keller C, Steensberg A, Helge JW, Pedersen BK, Saltin B, Neufer PD (2002) Influence of pre-exercise muscle glycogen content on exercise-induced transcriptional regulation of metabolic genes. J Physiol 541:261-271

Puigserver P, Rhee J, Lin J, Wu Z, Yoon JC, Zhang CY, Krauss S, Mootha VK, Lowell BB, Spiegelman BM (2001) Cytokine stimulation of energy expenditure through p38 MAP kinase activation of PPARgamma coactivator-1. Mol Cell 8:971-982

Randle PJ (1986) Fuel selection in animals. Biochem Soc Trans 14:799-806

Randle PJ, Newsholme EA, Garland PB (1964) Regulation of glucose uptake by muscle. 8 . Effects of fatty acids, ketone bodies and pyruvate, and of alloxan-diabetes and starvation, on the uptake and metabolic fate of glucose in rat heart and diaphragm muscles. Biochem J 93:652-665

Romijn JA, Coyle EF, Sidossis LS, Zhang XJ, Wolfe RR (1995) Relationship between fatty acid delivery and fatty acid oxidation during strenuous exercise. J Appl Physiol 79:1939-1945

Saleem A, Carter HN, Iqbal S, Hood DA (2011) Role of p53 within the regulatory network controlling muscle mitochondrial biogenesis. Exerc Sport Sci Rev 39:199-205

Steinberg GR, Watt MJ, McGee SL, Chan S, Hargreaves M, Febbraio MA, Stapleton D, Kemp BE (2006) Reduced glycogen availability is associated with increased AMPKalpha2 activity, nuclear AMPKalpha2 protein abundance, and GLUT4 mRNA expression in contracting human skeletal muscle. Appl Physiol Nutr Metab 31:302-312

Tunstall RJ, McAinch AJ, Hargreaves M, van Loon LJ, Cameron-Smith D (2007) Reduced plasma free fatty acid availability during exercise: effect on gene expression. Eur J Appl Physiol 99:485-493

Wojtaszewski JF, MacDonald C, Nielsen JN, Hellsten Y, Hardie DG, Kemp BE, Kiens B, Richter EA (2003) Regulation of 5'AMP-activated protein kinase activity and substrate utilization in exercising human skeletal muscle. Am J Physiol Endocrinol Metab 284:E813-E822 
Yeo WK, Paton CD, Garnham AP, Burke LM, Carey AL, Hawley JA (2008) Skeletal muscle adaptation and performance responses to once a day versus twice every second day endurance training regimens. J Appl Physiol 105:1462-1470

Yeo WK, McGee SL, Carey AL, Paton CD, Garnham AP, Hargreaves M, Hawley JA (2010) Acute signalling responses to intense endurance training commenced with low or normal muscle glycogen. Exp Physiol 95:351-358

Open Access This chapter is licensed under the terms of the Creative Commons Attribution 4.0 International License (http://creativecommons.org/licenses/by/4.0/), which permits use, sharing, adaptation, distribution and reproduction in any medium or format, as long as you give appropriate credit to the original author(s) and the source, provide a link to the Creative Commons license and indicate if changes were made.

The images or other third party material in this chapter are included in the chapter's Creative Commons license, unless indicated otherwise in a credit line to the material. If material is not included in the chapter's Creative Commons license and your intended use is not permitted by statutory regulation or exceeds the permitted use, you will need to obtain permission directly from the copyright holder.

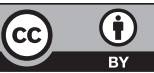

